\title{
When goals collide: Monitoring the goals of multiple characters
}

\author{
JOSEPH P. MAGLIANO \\ Northern Illinois University, DeKalb, Illinois \\ HOLLY A. TAYLOR \\ Tufts University, Medford, Massachusetts \\ and \\ HYUN-JEONG JOYCE KIM \\ Rhodes College, Memphis, Tennessee
}

\begin{abstract}
Most story plots contain multiple characters who are independent, interact, and often have conflicting goals. One would expect that narrative understanding would require monitoring of the goals, concerns, and situations of multiple agents. There is considerable evidence that understanders monitor the primary protagonist's goal plans (e.g., Suh \& Trabasso, 1993). However, there is relatively little research on the extent to which understanders monitor the goals of multiple agents. We investigated the impact of characters' roles and prominence on the extent to which understanders monitor the goal plans of multiple characters in a feature length film. In Experiment 1, participants made situation change judgments, and in Experiment 2, they verbally described scenes. Both types of judgments indicated that viewers monitor the goals and plans of multiple agents but do so to a greater extent for characters more prominent to the plotline.
\end{abstract}

Most stories contain multiple characters with their own knowledge states, emotions, and goals. At times, the goals of multiple characters can work in concert. More often than not, their conflict drives the plotline. This is certainly the case with respect to a story's protagonist and antagonist. For example, in the movie Star Trek II: The Wrath of Khan (Sallin \& Meyer, 1982; hereafter, referred to as Wrath of Khan), Captain Kirk (the protagonist) confronts an old nemesis, Khan (the antagonist), who has vowed to kill Kirk at all cost. During their first confrontation, Khan approaches Kirk's ship, the Enterprise, in a stolen Federation starship. Khan's goal is to deceive Kirk into thinking that the stolen ship is disabled long enough to position himself for maximum damage to the Enterprise. At the same time, Kirk has the goal of helping his disabled comrades and instructs his crew to contact the ship and offer aid. Presumably, understanding this scene requires one to monitor both Kirk's and Khan's goals and to realize that these goals conflict. It can be argued that the potential conflict between Kirk's and Khan's goals would be a primary source of the tension and suspense felt by the viewer.

The extent to which one understands and becomes engaged in a narrative may lead to the monitoring of

We thank Anne Britt, Keith Millis, David Rapp, and three reviewers for their helpful comments on earlier versions of the manuscript. Correspondence concerning this article should be addressed to J. P. Magliano, Department of Psychology, Northern Illinois University, DeKalb, IL 60115 (e-mail: jmagliano@niu.edu). multiple characters' goals and plans. There is considerable evidence that readers monitor and infer a single protagonist's goals (e.g., Long \& Golding, 1993; Lutz \& Radvansky, 1997; Magliano \& Radvansky, 2001; Suh \& Trabasso, 1993; Trabasso \& Suh, 1993). However, there is relatively little research that has assessed whether people monitor the goals or knowledge states of multiple characters (Graesser, Bowers, Olde, \& Pomeroy, 1999; Graesser, Bowers, Olde, White, \& Person, 1999; Richards \& Singer, 2001). In order to construct a coherent understanding of a narrative, understanders should be aware not only of the main character's (i.e., the protagonist's) knowledge states, but also of those of other characters, especially characters who are in conflict with the protagonist (i.e., the antagonists). When a main character has failed to achieve a goal, oftentimes this failure is the direct result of another character's goal-directed actions. In order to construct a coherent representation of a narrative, one must infer and represent the relationships between characters, their goals, and their actions. The purpose of this study was to determine whether people monitor the goal status of multiple characters when understanding a narrative film and whether specific features of characters, which will be discussed below, influence the likelihood of their being monitored. Because we know that people monitor the protagonist's goals, this character will serve as the baseline against which we will compare the monitoring of other characters.

Professionally produced films were used to examine our research question for several reasons. First, there is relatively little research on narrative understanding in the 
context of film (Baggett, 1979; Magliano, Dijkstra, \& Zwaan, 1996; Magliano, Miller, \& Zwaan, 2001; Schwan, Garsoffky, \& Hesse, 2000; Tan, 1996), as compared with that for narrative text. Theories and empirical findings derived from research on narrative texts should generalize to narrative film (Magliano et al., 2001). Second, professionally produced narratives provide a basis for identifying and examining naturally occurring but theoretically important factors that may influence the monitoring of the goals of multiple characters. The value of using naturalistic materials is that they provide ecological validity and results from these materials can then be used as a guide for future research with more traditional experimental approaches (see Magliano \& Graesser, 1991, for a similar argument). Finally, professionally produced narratives are engaging. It was critical that the participants be sufficiently engaged in the narrative experience that potentially, they might monitor multiple characters' goals. Without such engagement and/or motivation, the participants would have relatively low standards for understanding, leading to narrative representations that were minimally coherent (van den Broek, Risden, \& Husebye-Hartmann, 1995). Under such minimal comprehension strategies, we would not expect viewers to monitor the goals of a single character, much less multiple characters.

\section{Factors That Influence the Monitoring of a Character's Goals}

Simply stated, narratives are about characters interacting in a story world. In fact, some discourse-processing researchers have proposed that characters are the central component of situation models (Scott Rich \& Taylor, 2000) and of aesthetic and affective responses (Özyürek \& Trabasso, 1997; Zillmann, 1994). Supporting this assumption, Scott Rich and Taylor found that character shifts within a narrative led to greater decreases in (1) judgments of narrative coherence, (2) judgments of cohesion between narrative sentences, and (3) the accessibility of narrative entities than did shifts in either time or location. Trabasso (Özyürek \& Trabasso, 1997) and Zillmann have similar views on the role of characters in comprehension and affective responses (see also Allbritton \& Gerrig, 1991). Both of them assume that narrative involvement is heavily influenced by an evaluation of whether narrative events benefit or harm characters and, in particular, characters' goals. If an event benefits a sympathetic character or harms a disliked character, readers are pleased, whereas if the event harms a liked character or helps a disliked character, they respond negatively (Allbritton \& Gerrig, 1991; Özyürek \& Trabasso, 1997; Rapp \& Gerrig, 2002).

If characters do, indeed, play center stage in situation model construction and affective response, it is reasonable to assume that understanders will monitor the goals of multiple characters. However, working memory constraints most likely limit the number of characters that one can monitor in any given story (Graesser, Bowers, Olde, \& Pomeroy, 1999; Graesser, Bowers, Olde, White, \& Person, 1999). Several factors potentially mediate the extent to which we monitor a character's goals. In this initial study, we assessed two factors that are both readily apparent in narratives and relevant to theories of discourse processing and aesthetic response. The first factor involves the character's role, exemplified by the distinction between protagonist and antagonist. The second factor involves the character's centrality in the plotline. These factors will be discussed in turn.

The protagonist-antagonist distinction hinges upon goal conflict; these characters desire mutually exclusive states of being. For example, in Wrath of Khan, Captain Kirk wants to protect a top secret project and stay alive, whereas Khan wants to steal the project, exploit it as a weapon, and kill Kirk in the process. Protagonists are usually portrayed in a positive fashion, and thus, viewers may feel a sense of empathy with them (e.g., Zillmann, 1994). Many narratives contain a primary protagonist, who is the focus of the narration. In contrast, antagonists are usually portrayed in a negative fashion, and thus, viewers may feel a sense of counterempathy toward them (e.g., Zillmann, 1994). Conflicts in narratives are often introduced when the antagonists provide obstacles, often through their actions, to the protagonist's goals (Mandler \& Johnson, 1977; Thorndyke, 1977). In the present study, we established the protagonist as a character who has compelling positive goals in the narrative. The antagonist was the character(s) whose compellingly negative goals directly conflicted with those of the protagonist. The protagonist-antagonist distinction may be clearer for some genres than for others. We chose an action adventure and a science fiction film because they have well-defined protagonist-antagonist goal conflicts. Furthermore, in these genres, the morality of the protagonist and the antagonist are clear-cut.

It is possible that understanders monitor the protagonist's goals more closely than those of an antagonist. Theories of viewer/reader response in narratives provide a basis for this prediction (e.g., Metz, 1982). Literary theorists propose that understanders engage in a process of identification with the protagonist, but not with the antagonist (e.g., Metz, 1982), and because of this, narrative response is a vicarious experience of one's own "life" through the protagonist's "eyes" as the narrative unfolds. In essence, the viewer adopts the protagonist's goals.

According to an extreme view of role identification, understanders will experience the narrative world through the protagonist, and consequently, their understanding and memory will be entirely constrained by the protagonist's perspective. This strong view has been challenged in literary and film theory (e.g., Zillmann, 1994) and in psychology (Albrecht, O'Brien, Mason, \& Myers, 1995; Gerrig, 1996). The opposing view suggests that readers do not lose themselves in a character but, instead, build narrative representations that reflect the reader's perspective. For example, readers' knowledge of the story world is not constrained by the protagonist's knowledge states but, rather, is constrained by the nature of the information in prior discourse (e.g., how elaborated it is or how far back it is mentioned; Albrecht et al., 1995; Gerrig, 1996).

It is possible that a weaker form of identification operates during narrative understanding. According to this 
view, a reader's story representation is partially constrained by a character's perspective. In support of this position, there is some evidence that the availability of information in working memory is constrained by the deictic perspective of a protagonist (Black, Turner, \& Bower, 1979; Horton \& Rapp, 2003). For example, Horton and Rapp demonstrated that the availability of story objects waxed and waned as a function of whether those objects were in the protagonist's visual field. We expect that this would extend to the internal perspectives of protagonists, such as the character's goals (see also Özyürek \& Trabasso, 1997).

The second factor that may impact the monitoring of characters' goals is the centrality of that character in the storyline (Graesser, 1981; Graesser \& Clark, 1985; Myers \& O'Brien, 1998; Trabasso \& Nickels, 1992). Centrality refers to the extent to which a narrative entity is connected within the network representation of the narrative. Causality, including inferred relationships between initiating events, goals, attempts, and outcomes, plays a primary role in determining centrality and position in the hierarchical representation of a narrative (Graesser, 1981; Graesser \& Clark, 1985; Trabasso, van den Broek, \& Suh, 1989). There is a considerable amount of evidence supporting this assumption. Narrative entities and event that are central tend to be monitored more closely (Trabasso \& Nickels, 1992; Zwaan \& Radvansky, 1998), judged to be more important (e.g., Trabasso \& Sperry, 1985), and remembered better (e.g., Trabasso \& van den Broek, 1985) than are those that are not.

In the present study, we distinguish between primary and secondary characters that vary in centrality to the plotline. Primary characters are central to the story plotline from the time they are introduced, usually during the opening scenes (Mandler \& Johnson, 1977; Thorndyke, 1977), and are involved in longer causal chains than are secondary characters (Trabasso \& Nickels, 1992). For this reason, events that happen to primary characters and their ensuing actions move the story along. Secondary characters are transitory to the plotline, appearing and interacting in only a few scenes. As such, they will have short causal chains associated with them.

One obvious prediction is that understanders will monitor the goals and plans of primary characters more closely than those of secondary characters. In support of this prediction, Graesser and his colleagues have shown that a narrative agent's salience in the discourse mediates the extent to which readers monitor their knowledge states (Graesser, Bowers, Olde, White, \& Person, 1999) and remember their dialogue (Graesser, Bowers, Olde, \& Pomeroy, 1999). Although Graesser's research is relevant to the present study and this prediction, it is important to note that they did not study character goals.

Character role and centrality are clearly separate factors but are certainly not mutually exclusive. For example, the main protagonist, by definition, will be the most central character. In fact, Trabasso and Nickels (1992) defined the protagonist as the character with the longest causal chain, on the basis of a causal network analysis of the story. Furthermore, there is a preponderance of research suggesting that viewers monitor the goals of the primary protagonist (e.g., Long \& Golding, 1993; Lutz \& Radvansky, 1997; Magliano \& Radvansky, 2001; Suh \& Trabasso, 1993). Thus, we conceptualized this character as a baseline control. Specifically, we compared the extent to which viewers monitor the goal episodes of an antagonist(s) as a function of the centrality of that character(s) in a film.

\section{Overview of the Present Study}

As was suggested in the last section, it would be challenging to find real films that completely crossed character role (protagonist vs. antagonist) with character centrality (primary vs. secondary) while also controlling for other character differences. In fact, it would be challenging to write experimentally generated materials that would fully cross these variables, be free of confounds, and be sufficiently engaging (see Magliano \& Graesser, 1991, for an extended argument). Thus, we chose films that afforded interesting contrasts between the types of characters exemplified by these two variables. For the reasons given above, we concluded that contrasting the primary protagonist (or baseline character) with both primary and secondary antagonists would be interesting. The James Bond movie Moonraker (Broccoli \& Gilbert, 1979) allows a contrast between a primary protagonist and secondary antagonist(s). In this film, James Bond interacts with and disposes of several secondary antagonists, who usually appear in only one or two scenes. As such, these secondary antagonists have relatively short causal chains associated with them in the story representation and have relatively low centrality in the plotline. In contrast, Wrath of Khan allows a contrast between a primary protagonist (Kirk) and a primary antagonist (Khan). The plotline progresses through successive interactions between these characters. Khan has a longer causal chain than do the Moonraker antagonists and is definitely more central in the plotline as a result. We examined the extent to which viewers monitored the goal plans of the protagonist and antagonist(s) in scenes in which the characters interacted. In these scenes, the goals of the protagonist and the antagonist are in direct conflict. Again, the comparison of interest is the extent to which viewers monitor the antagonists as a function of their centrality in the plotline, with the primary protagonist being used as a baseline.

We adopted an approach similar to one used by Zacks, Tversky, and Iyer (2001), which was based on seminal research by Newtson (1973) on event partonomy. Zacks et al. (2001) relied on corroborating data from an event partonomy task and event descriptions to investigate the monitoring and understanding of activities. In an event partonomy task, participants watch a videotape of people performing familiar activities (e.g., washing the dishes) or unfamiliar ones (e.g., assembling a saxophone). Their task is to segment the activity into discrete actions, indicating when actions change by pressing a button. In Zacks et al. (2001), participants described the events after viewing the 
videotapes. The researchers found convergence between the data sets, suggesting that the people understood the activities as consisting of hierarchically related actions. Specifically, the participants came up with the same discrete actions in both data sets.

Experiment 1 of the present study constituted a reanalysis of the data collected by Magliano et al. (2001), who adopted a modified event partonomy paradigm. In that study, Magliano et al. assessed the extent to which viewers monitor temporal and spatial shifts in films. Participants viewed a feature length narrative film. They made situation change judgments, in which they identified points that contained a change in the situation or circumstances that the characters were facing. The participants were not given any instructions as to what factors might constitute a situation change. Magliano et al. (2001) found that these judgments corresponded to shifts in time and space within the narrative world, as indicated by cinematic device. In Experiment 2, we collected event descriptions as participants viewed a movie. As occurred in Zacks et al. (2001), we expected convergence between the event descriptions and the situation change data. In Experiment 1, we were interested in assessing whether the situation change judgments corresponded to shifts in the goal episodes associated with the protagonist or the antagonist. The details of how we assessed these shifts will be discussed below. With respect to Experiment 2, we were interested in assessing the extent to which viewers mention characters in their event descriptions.

An identification hypothesis predicts that viewers will monitor a character's goals primarily as a function of that character's role, rather than as a function of centrality in the narrative (Metz, 1982). According to this hypothesis, change judgments will increase for shifts in the goal episodes associated with the primary protagonist, but not for those of antagonists, regardless of their centrality (i.e., regardless of which film is watched). Furthermore, participants will talk more about the protagonist than about the antagonist, regardless of character centrality. This is based on the assumption that viewers will identify more closely with the protagonists than with the antagonists, motivating them to monitor primarily their goals in the plotlines.

A centrality hypothesis predicts that viewers will monitor a character's goals if that character is prominent, regardless of role. Specifically, this hypothesis would predict that situation change judgments should increase when there are shifts in the goal episodes for the primary protagonists in both films in the present study and for the primary antagonist (i.e., Khan in Wrath of Khan), but not for secondary antagonists (i.e., those in Moonraker). Furthermore, participants will talk about primary characters more than about secondary characters when describing story events.

\section{EXPERIMENT 1}

If viewers are monitoring the status of a character's goals, their sense of the ongoing situations should be influenced by major shifts in the causal episodes for those characters.
Participants watched either Moonraker or Wrath of Khan. Moonraker provided a contrast between a primary protagonist and several secondary antagonists; Wrath of Khan provided a contrast between a primary protagonist and a primary antagonist. In Experiment 1, we assessed whether situation change judgments increase as a function of goal episode shifts associated with a character. The causal network model (Trabasso et al., 1989) provided the basis for understanding a causal episode's components. According to the causal network model, story events can be classified according to how they fit into an episodic structure. Episodes consist of a set of story unit categories (Stein \& Glenn, 1979). These categories include settings, events, goals, attempts, outcomes, and reactions. Setting information refers to a story's time and place. Events are experienced by a character but are not the direct result of his/her actions. Goals relate to a character's desired state of the world. Attempts are a character's overt actions to achieve some desired state. Outcomes result from a character's actions. Finally, reactions refer to a character's emotional responses or changes in knowledge state. The model assumes inherent causal relationships between these elements. For example, initiation events cause psychological changes that lead to the formation of goals. These goals causally motivate a character to take action. Finally, these actions lead to outcomes that are either the desired state or a goal failure (i.e., some state of affairs other than the desired one). We assume that if viewers are closely monitoring a character's goals, they should be aware of major shifts in goal episodes, because the shifts have causal implications for the character's goals (Trabasso et al., 1989).

According to the causal network model and story grammar theory in general (Stein \& Glenn, 1979), narrative plots and scenes are structured around a hierarchy of goal episodes involving a society of characters. For example, in the scene described at the beginning of this article, Kirk experiences an initiating event when the Reliant, Khan's ship, approaches the Enterprise. In response, Kirk has the goal of finding out what the Reliant wants. He attempts to achieve this goal by ordering his communications officer to contact the Reliant. The outcome of this attempt is that the Reliant responds that their communication system is down and they need help. On the other hand, Khan has two explicitly stated superordinate goals, to kill Kirk and to find information on a top-secret project called Genesis. He has a subgoal of deceiving Kirk into thinking that the Reliant is disabled and attempts to achieve this goal by responding with a distress signal to Kirk's communication attempt.

In all of the scenes examined in this study, the protagonist and the antagonist goal episodes are in direct conflict. Expert raters (the first author and a research assistant) analyzed each scene from the perspectives of both the protagonist and the antagonist, identifying camera shots within a scene that depicted the initiation events, the initial actions associated with an attempt to achieve a salient goal, and the end of the action sequence associated with a salient goal for both characters. Trabasso and his colleagues conducted a similar analysis of story description 
protocols in order to assess shifts in character perspective that children and adults adopted when narrating picture stories (Trabasso \& Nickels, 1992). The rules for making these decisions are described in the Method section.

The results of this discourse analysis were used to predict situation change judgments in a series of multiple regression analyses. If viewers are closely monitoring the goal episodes of a character, situation judgments should increase as a function of all three shifts in the causal episodes. Furthermore, one would expect the percentage of the variance accounted for by the predictor variables to reflect how closely the viewers were monitoring a character's goals. According to the strong identification hypothesis, the predictor variables associated with the antagonist will not significantly predict the situation change judgments, and the variance explained by these variables will be considerably less than that explained by those associated with the protagonist. According to the centrality hypothesis, the pattern of significance and the variance explained will be comparable for the primary protagonist and the primary antagonist, but not for the secondary antagonist.

\section{Method}

Participants. Forty undergraduates at Northern Illinois University participated for course credit.

Materials. The participants viewed one of two films. Moonraker is a James Bond movie classified in the action adventure genre. Wrath of Khan is the second of the Star Trek film series and is classified in the science fiction genre. Moonraker allowed a contrast between a primary protagonist and secondary antagonist(s), and Wrath of Khan allowed a contrast between a primary protagonist and a primary antagonist. Twenty participants viewed each film.

Discourse analyses. Eight scenes from Moonraker and five scenes from Wrath of Khan were chosen for discourse analyses. These scenes were chosen because they depicted the protagonist and the antagonist(s) interacting with one another. A discourse analysis was then conducted to determine the camera shots that depicted changes in the goal episodes, from the perspective of either the protagonist or the antagonist. In order to conduct this film segment analysis, the individual shots that made up the segments were identified. A shot was defined as a continuous piece of film from one camera angle, without breaks in the continuity of action. There were 470 and 340 shots in the segments for Moonraker and Wrath of Khan, respectively. The average shot lengths were 3.06 and $4.77 \mathrm{sec}$ for Moonraker and Wrath of Khan, respectively.

For each scene, the first author and a research assistant constructed a verbal description of the primary goals and plans of both the protagonist and the antagonist. In identifying these goal episodes, the raters determined the salient superordinate and subordinate goals of both characters in a scene. These scene descriptions were then used to analyze the specific shots making up the scenes. From the perspective of each character, the raters determined whether a shot depicted an initiating event, the beginning of an action sequence, or the end of an action sequence. A shot sequence was coded as an initiating event if it depicted an unambiguously recognized event happening to a character that had causal significance on future behaviors. Shots judged as depicting initiating events were assigned a 1 , and all other shots were assigned a 0 for this variable. A shot was judged as depicting the beginning of an action sequence if it contained either the first verbal or the first behavioral action associated with a superordinate or a subordinate goal. If the shot was the beginning of a superordinate goal plan, it was assigned a 2, and if it was the beginning of a subordinate goal plan, it was assigned a 1 . All other shots were assigned a 0 for this variable. A shot was determined to be the end of an action sequence if it depicted either the successful or the unsuccessful completion of a superordinate or a subordinate goal. If the shot was the end of a superordinate goal plan, it was assigned a 2, and if it was the end of a subordinate goal plan, it was assigned a 1 . All other shots were assigned a 0 for this variable. Table 1 contains the number of shots in each of these categories for each type of character for each film.

Procedure. In the present study, we used data collected by Magliano et al. (2001), who modified Newtson's (1973) event partonomy task. The participants viewed one of the two films. Before viewing the film, they were told that the researchers were interested in how people understand the situations that arise in a movie. They were told to identify points in the film at which the circumstances or situation changed. They were to consider events that occurred at any point in time when making these decisions. However, no specific instructions regarding what would constitute a change in situation or circumstances were given. The instructions were intentionally vague so as not to bias the participants' intuitive understanding of what constitutes a change in a narrative situation. The participants were given a remote control for the VCR and were instructed to keep their finger on the pause button throughout the film. They were to pause the film whenever they thought the situation or circumstance had changed. A videotape for each film included a running time counter, accurate up to a 10th of a second. After pausing the film, the participants wrote down the time indicated on the screen and then continued the videotape.

\section{Results and Discussion}

A series of multiple regression analyses were performed on situation change scores for each film separately. The shots that made up the film segments were the units of analysis. A situation change score was calculated for each shot by dividing the number of participants who made a change judgment for a given shot by the total number of participants viewing the film. There were six predictor variables for each analysis: initiating events, beginnings of action sequences, and ends of action sequences for both the protagonist and the antagonist. Hierarchical regression analyses were conducted in order to determine the

Table 1

Numbers of Initiating Event, Beginning of Action Sequence, and End of Action Sequence Shots as a Function of Character and Film

\begin{tabular}{lccccc}
\hline & \multicolumn{2}{c}{ Moonraker } & & \multicolumn{2}{c}{$\begin{array}{c}\text { Star Trek II: The Wrath } \\
\text { of Khan }\end{array}$} \\
\cline { 2 - 3 } \cline { 5 - 6 } $\begin{array}{l}\text { Predictor } \\
\text { Variable }\end{array}$ & Protagonist & $\begin{array}{c}\text { Secondary } \\
\text { Antagonist }\end{array}$ & & Protagonist & $\begin{array}{c}\text { Primary } \\
\text { Antagonist }\end{array}$ \\
\hline Initiating event & 33 & 14 & & 42 & 25 \\
Beginning & 32 & 27 & & 20 & 16 \\
Ending & 35 & 25 & & 16 & 13 \\
\hline
\end{tabular}


unique variance accounted for by the variables associated with a given character. In order to determine the amount of unique variance accounted for by the variables associated with the protagonist, the variables associated with the antagonist were force entered into the regression equation in the first step. The variables associated with the protagonist were forced entered in a second step, and variance explained was calculated. The opposite procedure was used to estimate the unique variance accounted for by the variables associated with the antagonists.

Table 2 contains the beta weights and variance accounted for by the variables associated with the protagonist and the antagonist for each film. With respect to the protagonist in Moonraker, situation change scores increased as a function of shots containing initiating events $[t(463)=5.14$, $p<.05]$, beginnings of action sequences $[t(463)=6.19$, $p<.05]$, and ends of action sequences $[t(463)=7.40, p<$ $.05]$. Conversely, for the antagonists, only the beginningof-action-sequence variable predicted change judgments $[t(463)=10.47, p<.05]$.

With respect to the protagonist in Wrath of Khan, situation change scores increased as a function of shots containing initiating events $[t(339)=2.87, p<.05]$, beginnings of action sequences $[t(339)=3.07, p<.05]$, and ends of action sequences $[t(339)=2.21, p<.05]$. Similarly for the antagonist, situation change scores increased as a function of shots containing initiating events $[t(339)=3.30$, $p<.05]$, beginnings of action sequences $[t(339)=2.19$, $p<.05]$, and ends of action sequences $[t(339)=2.92, p<$ $.05]$.

The results of this experiment suggest that the viewers monitored the goal plans of multiple agents when engaged in understanding a narrative film. However, the extent to which the viewers monitored a character's goals was contingent upon character prominence, rather than upon role. This interpretation is based primarily on the pattern of significance in the beta weights for the predictor variables. In particular, only the beginnings of action sequences were predictive of change judgments for the secondary antagonist, whereas initiating events, beginnings of action sequences, and ends of action sequences predicted change judgments for both the primary protagonist and the antagonist.

These results are consistent with the centrality hypothesis and suggest that understanders most closely monitor and index central entities in a narrative (e.g., Graesser,
1981). Specifically, shifts in the goal episode for the primary protagonists increased the likelihood of situation change judgments for both films. However, the centrality of the antagonist mediated the extent to which these shifts increased change judgments. Specifically, episode shifts were monitored more closely for the primary antagonist than for the secondary antagonists. Interestingly, variables associated with the protagonist and antagonists in Moonraker accounted for a comparable amount of the variance: $17 \%$ and $15 \%$, respectively, whereas the centrality hypothesis predicts that the variance explained should be less for this film's antagonists than for the protagonist. We believe that this was the case because the antagonists' initial attempts had the greatest impact on the well-being of the protagonist and, as such, took on great import for the viewer.

\section{EXPERIMENT 2}

The goal of Experiment 2 was to further validate the results of Experiment 1, which suggested that understanders monitor the central character's goals, irrespective of role. Specifically, we collected event descriptions as viewers watched the same scenes as those analyzed in Experiment 1 . The event descriptions for Moonraker came from Magliano et al. (1996). They had viewers think aloud while watching at several locations in the film. A subset of these locations, which occurred during the scenes analyzed in Experiment 1, was used for this experiment. New protocols were collected for Wrath of Khan, using the same think-aloud instructions. We assessed the extent to which the characters were described as a function of character type and prominence (i.e., as a function of film). According to the identification hypothesis, participants will describe the protagonist to a greater extent than they will the antagonists, regardless of the character's prominence. According to the centrality hypothesis, we expected that the participants who watched Moonraker would describe the goal plans of the protagonist to a greater extent than they would those of the antagonists, whereas the participants who watched Wrath of Khan would describe the goal plans of the protagonist and the antagonist equally. These results would also be consistent with those of Experiment 1 .

We also assessed the nature of the character descriptions. To do so, we parsed event descriptions into clauses

Table 2

Beta Weights and Variance Explained From the Regression Analyses on the Situation Change Scores

\begin{tabular}{|c|c|c|c|c|}
\hline \multirow[b]{2}{*}{$\begin{array}{l}\text { Predictor } \\
\text { Variable }\end{array}$} & \multicolumn{2}{|c|}{ Moonraker } & \multicolumn{2}{|c|}{$\begin{array}{c}\text { Star Trek II: The Wrath } \\
\text { of Khan }\end{array}$} \\
\hline & Protagonist & $\begin{array}{l}\text { Secondary } \\
\text { Antagonist }\end{array}$ & Protagonist & $\begin{array}{c}\text { Primary } \\
\text { Antagonist }\end{array}$ \\
\hline Initiating event & $.19^{*}$ & .03 & $.15^{*}$ & $.17^{*}$ \\
\hline Beginning & $.28^{*}$ & $.38^{*}$ & $.16^{*}$ & $.11^{*}$ \\
\hline Ending & $.28^{*}$ & .05 & $.12^{*}$ & $.15^{*}$ \\
\hline Variance explained & $17 \%{ }^{*}$ & $15 \% *$ & $15 \% *$ & $14 \% *$ \\
\hline
\end{tabular}


containing main verbs. Table 3 contains example protocols for Moonraker (Participants 1 and 2) and Wrath of Khan (Participants 3 and 4), which will be used to illustrate the coding scheme. We developed a clause-based coding scheme, loosely based on case role grammars (e.g., Fillmore, 1968, 1972). Specifically, we determined whether a character was described as an actor, a recipient, or an internal reactor. An additional classification was added for clauses that were descriptive of a character. A character was considered an actor when he/she was performing an action, having a goal, or achieving an outcome. For example, in Clause 1 from Participant 1, the protagonist was described as an actor, because the clause conveyed Bond's action. In Clause 3, the protagonist was described as an actor, because the clause conveyed the outcome of Bond's attempt to escape his pursuers (e.g., he is success$f u l)$. The second clauses from both Participants 1 and 2 were coded as describing the antagonist as an actor, because they conveyed the goal of the enemy agents (i.e., they want to kill Bond). A character was judged to be a recipient if he/she was experiencing another character's action, was the object of another character's goal or emotional state, or experienced an unintentional event (e.g., a thunderstorm). For example, the protagonist was judged to be a recipient of the antagonist's goal in Clause 2 from both Participants 1 and 2. In Clause 4 from Participant 4, the protagonist was judged to be a recipient, because he was the object of the antagonist's emotional response. A character was judged to be internally reacting if he/she was experiencing an emotional state (e.g., anger or surprise) or experiencing an emerging knowledge state (e.g., realizing or recognizing). For example, the antagonist in Clause 4 from Participant 4 was judged to be a reactor, because the clause described his emotional attitude toward the protagonist (e.g., Khan hates Kirk). Finally, a clause was labeled as descriptive if it mentioned the character's physical or knowledge state. For example, Clause 2 from Participant 4 concerning the protagonist and Clause 3, also from Participant 4, concerning the antagonist were judged as descriptive, because the clauses indicated the spatial locations of Kirk and Khan, respectively.

Within the coding scheme, a character could be explicitly or implicitly mentioned. Explicit mentions described characters by name (e.g., Kirk or Khan), in a noun phrase (e.g., the Japanese guy), or via a pronoun. We adopted a strong criterion for indexing a character, so that characters referenced with a plural pronoun were not considered. For example, even though Kirk is part of the group referenced in Clauses 5-7 from Participant 4, he was not judged as having been explicitly referenced. A character was judged to be implicitly mentioned if the clause was a subordinate clause and the character was explicitly mentioned in an earlier or later clause. For example, Clauses 5 and 6 from Participant 1 were judged to reference actions of Bond, who had been explicitly mentioned in Clauses 3 and 4 . The first and second authors coded the protocols and had a high interrater reliability $(K=.89)$.

According to an identification hypothesis, participants will describe the initiating events goals, actions, and outcomes of a protagonist to a greater extent than those of an antagonist(s). Thus, the protagonist will be described in each of the roles more often than will the antagonist(s), regardless of film (centrality). This may seem counterintuitive, because the antagonist should be the recipient of many of the protagonist's actions, whereas the protagonist is the recipient of the antagonist's actions. However, it is possible to discuss a protagonist as a recipient of an action without mentioning the actor (e.g., Bond is being

Table 3

Example Event Descriptions From Experiment 2

\begin{tabular}{|c|c|c|c|c|c|c|c|c|c|}
\hline \multirow[b]{2}{*}{ Part. } & \multirow[b]{2}{*}{ Clause } & \multicolumn{4}{|c|}{ Protagonist } & \multicolumn{4}{|c|}{ Antagonist } \\
\hline & & A & Recip. & React. & $\mathrm{D}$ & A & Recip. & React. & $\mathrm{D}$ \\
\hline 1 & 1. He's on the run again & $\mathrm{x}$ & & & & & & & \\
\hline 1 & 2. from new people trying to kill him. & & $\mathrm{x}$ & & & $\mathrm{x}$ & & & \\
\hline 1 & 3. Bond is so far successful, but & $\mathrm{x}$ & & & & & & & \\
\hline 1 & 4. he just ran into a red light and & $\mathrm{x}$ & & & & & & & \\
\hline 1 & 5. will either narrowly escape again or & $\mathrm{x}$ & & & & & & & \\
\hline 1 & 6. cause an accident. & $\mathrm{x}$ & & & & & & & \\
\hline 2 & 1. Bond is trying to escape from men & $\mathrm{x}$ & & & & & $\mathrm{x}$ & & \\
\hline 2 & 2. who obviously want him dead, but & & $\mathrm{x}$ & & & $\mathrm{x}$ & & & \\
\hline 2 & 3. Bond is going to crash into another boat & $\mathrm{x}$ & & & & & & & \\
\hline 2 & 4. that has two lovers in it. & & & & & & & & \\
\hline 3 & 1. Khan and Kirk are about to encounter. & $\mathrm{x}$ & & & & $\mathrm{x}$ & & & \\
\hline 3 & 2. We learned what Genesis is. & & & & & & & & \\
\hline 3 & 3. Khan plans on attacking the Enterprise. & & & & & $\mathrm{x}$ & & & \\
\hline 3 & 4. Kirk is not following protocol. & $\mathrm{x}$ & & & & & & & \\
\hline 4 & 1. Both ships are going toward Space Lab. & & & & & & & & \\
\hline 4 & 2. The one has the Admiral, and & & & & $\mathrm{x}$ & & & & \\
\hline 4 & 3. the other one has the guy & & & & & & & & $\mathrm{x}$ \\
\hline 4 & 4. that hates him. & & $\mathrm{x}$ & & & & & $\mathrm{x}$ & \\
\hline 4 & 5. Enterprise has seen the other ship & & & & & & & & \\
\hline 4 & 6. and they know it's Reliant. & & & & & & & & \\
\hline 4 & 7. They are wondering what they are doing. & & & & & & & & \\
\hline
\end{tabular}

Note_-Part., participant; A, actor; Recip., recipient; React., reactor; D, descriptive. 
attacked, but I'm sure he'll escape). Furthermore, an antagonist's initial actions often serve as initiation events for the protagonist, and once the initiating event is mentioned, the goals and plans of the protagonist will become the focus of attention. According to a centrality hypothesis, primary characters will be described in each role more often than secondary characters. Thus, there will be an interaction between film and role description. Specifically, the protagonist in Moonraker would be described in each role more often than the secondary antagonists. On the other hand, the protagonist and the antagonist should be described in the different roles at about the same frequency for Wrath of Khan.

\section{Method}

Participants. Ten of the participants were undergraduates at the University of Chicago and participated for $\$ 10$. Ten of the participants were from Northern Illinois University and participated for course credit.

Materials. The participants watched either Moonraker or Wrath of Khan in its entirety. The participants described the ongoing events for the same scenes as those that were analyzed in Experiment 1: 13 locations in Moonraker and 15 locations in Wrath of Khan. The selected scenes emphasized interactions between the protagonist and the antagonist(s). In order to provide practice in producing event descriptions, the participants produced eight descriptions for Moonraker and seven for Wrath of Khan before the first experimental location.

Procedure. The participants watched the films in groups. They were told that the purpose of the study was to examine how people understand movies and were instructed to write about their understanding each time the movie was paused. The participants were told to write whatever came to mind and to focus their thoughts on the current scene, rather than on prior scenes. They were given as much time as needed to write. These pauses typically lasted between 1 and $3 \mathrm{~min}$.

\section{Results and Discussion}

The first analysis was conducted to determine the proportion of clauses that explicitly or implicitly indexed the protagonist or antagonist as a function of which film the participant viewed. Character type was a withinparticipants variable, whereas film was a betweenparticipants variable. A $2 \times 2$ ANOVA revealed a main effect of character in which protagonists $(M=.48, S D=$ .12) were indexed more frequently than antagonists $[M=$ $.37, S D=.11 ; F(18)=27.303, M S_{\mathrm{e}}=0.004648, p<$ $.01]$. This main effect was qualified by a character type $\times$ film interaction $\left[F(18)=48.627, M S_{\mathrm{e}}=0.004648, p<\right.$ $.01]$. A Tukey post hoc analysis revealed that the protago- nist $(M=.57, S D=.08)$ was indexed more frequently than the antagonist $(M=.31, S D=.08)$ in Moonraker, whereas the protagonist $(M=.39, S D=.08)$ and the antagonist $(M=.43, S D=.10)$ were mentioned at the same frequency in Wrath of Khan.

A second analysis was conducted to determine whether characters were described differently as a function of film. Specifically, a 2 film (Moonraker vs. Wrath of Khan) $\times 2$ character type (protagonist vs. antagonist) $\times 4$ case role (actor, recipient, reactor, or descriptive) mixed ANOVA was conducted on the proportion of clauses. Table 4 contains the mean proportions for this analysis. We report only main effects and interactions that involved case role, which was the unique contribution of this analysis. There was a main effect of case role $\left[F(18)=301.44, M S_{\mathrm{e}}=\right.$ $0.00147, p<.05]$. A Tukey post hoc test revealed that characters were mentioned more often as actors $(M=.24)$ than as recipients $(M=.13)$, which, in turn, were mentioned more often than character descriptions were $(M=$ $.05)$, which were mentioned more often than reactors $(M=$ $.01)$. There was a significant interaction between character type and case role $\left[F(18)=28.58, M S_{\mathrm{e}}=0.001395, p<\right.$ $.05]$. More important, there was a significant film $\times$ character type $\times$ case role interaction $\left[F(18)=4.33, M S_{\mathrm{e}}=\right.$ $0.001395, p=.052]$. A Tukey post hoc analysis revealed that the protagonist was indexed more often as an actor and a recipient than were the antagonists in Moonraker. There were no differences in the frequency of indexing the protagonist and the antagonists as reactors or of giving descriptives. On the other hand, there were no significant differences in the frequency of indexing the protagonist or the antagonist in the different case roles for Wrath of Khan.

These results suggest that the viewers monitored the goal plans of the characters that were more central to the storyline (primary characters) more than they did those of the secondary characters. As such, these results are most consistent with the centrality hypothesis. This interpretation of the event description data is bolstered by their convergence with the situation change judgment data.

The analysis of the case role data indicated that central characters were described more as actors and recipients, with an orientation more toward actors. This may stem from the fact that these case roles map onto the major elements of the goal episodes for a character. Specifically, a character is typically the recipient of an initiating event, which may or may not be the result of another character's

Table 4

Mean Proportions of Clauses (With Standard Deviations) as a Function of Film, Character Type, and Case Role

\begin{tabular}{|c|c|c|c|c|c|c|c|c|c|}
\hline \multirow[b]{3}{*}{ Film } & \multirow[b]{3}{*}{ Character Type } & \multicolumn{8}{|c|}{ Case Role } \\
\hline & & \multicolumn{2}{|c|}{ Actor } & \multicolumn{2}{|c|}{ Recipient } & \multicolumn{2}{|c|}{ Reactor } & \multicolumn{2}{|c|}{ Described } \\
\hline & & $M$ & $\overline{S D}$ & $M$ & $S D$ & $M$ & $S D$ & $M$ & $S D$ \\
\hline \multirow[t]{2}{*}{ Moonraker } & Protagonist & .30 & .06 & .19 & .05 & .01 & .02 & .06 & .04 \\
\hline & Antagonist & .18 & .04 & .08 & .04 & .003 & .004 & .04 & .02 \\
\hline \multirow[t]{2}{*}{ Wrath of Khan } & Protagonist & .26 & .06 & .10 & .06 & .002 & .004 & .03 & .02 \\
\hline & Antagonist & .22 & .06 & .15 & .06 & .02 & .01 & .05 & .04 \\
\hline
\end{tabular}


intentional actions. Furthermore, the actor role reflects several elements of the goal episode, such as goals, actions, and outcomes. This may be one reason why this case role was the most prevalent for all character types. The prevalence of the actor case role is also consistent with theories of discourse comprehension that assume that readers direct their attention primarily to character goals and actions in a narrative (e.g., Graesser, 1981; Graesser \& Clark, 1985; Schank \& Abelson, 1977).

\section{GENERAL DISCUSSION}

Despite the fact that most narratives contain multiple characters interacting in a story world, relatively little research has been done that assesses the extent to which understanders monitor the concerns and construct elaborate representations of multiple characters (see Graesser, Bowers, Olde, \& Pomeroy, 1999; Graesser, Bowers, Olde, White, \& Person, 1999; Trabasso \& Nickels, 1992). Although it seems reasonable that narrative understanding and appreciation require viewers and readers to keep track of multiple characters' goals, it is also likely that working memory constraints place limitations on one's ability to do so. Certainly, one would not compute the internal states and goals of every narrative agent encountered. Therefore, it is important to assess the factors mediating the extent to which understanders monitor character goals.

In this research, we assessed the impact of a character's role and centrality in the narrative on the extent to which viewers monitor changes in that character's goals and plans. The results of both experiments suggest that character prominence impacts character monitoring more so than does character role. That is, there was a convergence between the pattern of beta weights for the situation change judgments and the mentioning of characters in the event descriptions, indicating that viewers monitored a primary antagonist's goals as closely as those of the primary protagonist but did not do so for secondary antagonists. To our knowledge, this is one of the first studies to illustrate that understanders monitor the goals of multiple agents, and it is certainly the first study to address this issue in the context of film. This research is consistent with the centrality hypothesis and with a large body of research in support of it in the context of narrative text (e.g., Graesser, 1981).

The results of this experiment are also consistent with Zillmann's (1994; de Wied, Zillmann, \& Ordman, 1994) theory of viewer response. Zillmann views the protagonistantagonist distinction as one of goal conflict. He argues that viewers monitor both types of characters. However, the extent to which one monitors a character's concerns is contingent on the strength of empathy or counterempathy. Thus, viewers closely monitor characters for whom they care strongly or against whom they have strong feelings. Viewers have a positive response when things go well for the protagonist and poorly for the antagonist. Conversely, they respond negatively when things go badly for the protagonist but well for the antagonist. The present study suggests that the degree of these affective responses may be mediated in part by a character's prominence. Clearly, more research in which this possibility is explored is warranted.

The present results are relevant to models of discourse understanding and situation model construction. The event-indexing model assumes that understanders monitor changes in multiple dimensions of situational continuity, such as characters, goals, causality, time, and space (Magliano, Zwaan, \& Graesser, 1999; Zwaan, Langston, \& Graesser, 1995; Zwaan \& Radvansky, 1998). It has been proposed that situation models are structured around the protagonist (Scott Rich \& Taylor, 2000), which then serves as the predominant situation model component. However, the event-indexing model does not address whether understanders monitor the goals and plans of multiple characters. The present study suggests that theories of situation model construction, such as the event-indexing model, should be modified to account for the monitoring of the goals of multiple characters.

Furthermore, the construct of centrality has a strong foundation in several theories of discourse comprehension, such as the constructionist (e.g., Graesser, 1981; Graesser, Singer, \& Trabasso, 1994; Trabasso et al., 1989) and resonance (e.g., Myers \& O’Brien, 1998) theories. To our knowledge, no other studies have addressed the role of centrality in monitoring the goals of multiple agents. The primary/secondary character distinction provided a viable way to operationalize this construct for naturalistic materials.

There is at least one limitation of the present study to the possible generalizability of these results to other narrative films. Specifically, we used only two films. Even so, there are several reasons to believe that our results will generalize to other narratives. First, both films are typical of the action adventure/science fiction genres. For example, both of them have salient protagonists and antagonists who are in goal conflict and have morally divergent goals. In addition, like other films in a variety of genres, the present films contain a society of characters that vary in centrality. Finally, the respective filmmakers adhered to the stereotypical techniques and practices of Hollywoodstyle films, as have been outlined by Bordwell (1985; see Magliano et al., 2001, for a discourse analysis of these with respect to films on the basis of Bordwell's analysis).

In addition, data collected by Magliano et al. (2001) afforded further support and better generalization through an analysis of another film. Specifically, we reanalyzed situation change judgments made by participants viewing Jeremiah Johnson (Coonan, Moder, Wizan, \& Pollack, 1972), a film that includes primary and secondary protagonists. This film portrays the life of Jeremiah Johnson, who meets and interacts with a series of secondary protagonists who are a part of his life until either he moves to a new location, these characters die, or both. In accord with Experiment 1, we conducted hierarchical multiple regression analyses on the situation change judgments. For the primary protagonist, initiating events, the beginning of action sequences, and the end of action sequences each significantly predicted an increase in situation change scores and accounted for a significant $17 \%$ of the 
variance $[F(3,802)=37.76, p<.01]$. For the secondary protagonist, initiation events and the beginning of action sequences predicted an increase in situation change judgments but accounted only for $4 \%$ of the variance $[F(3,802)=14.88, p<.01]$. These data further support the conclusion that primary characters are more closely monitored than secondary characters.

Another limitation of using naturalistic films is the lack of precise control over content. Other factors that covary with centrality should also influence the extent to which viewers monitor character goals. One such factor is foregrounding, which refers to the extent to which an entity is in the discourse focus. There is considerable evidence that foregrounded narrative entities and events are more available and are remembered better than backgrounded ones (e.g., Albrecht et al., 1995; Morrow, 1985). Indeed, filmmakers adopt techniques to foreground characters and implied internal states. Specifically, filmmakers use visual and sound-reproducing devices that invite the viewer to "see" the story world from the character's perspective. One such device is the point-of-view shot sequence, which consists of a close-up of a character's face, followed by the shot of a story event at about the character's eye level (Bordwell \& Thompson, 1993). It has been argued that such shot sequences encourage the viewer to infer the character's internal states, including emotions, beliefs, and goals, in the context of the unfolding events (Holland, 1992). One would expect that filmmakers use these devices more often in association with primary characters than with secondary characters. Clearly, further research is warranted to identify and assess other factors, such as foregrounding, that influence goal monitoring.

A final argument for the importance of the present research is the small but growing body of research on narrative film. The paucity of research on this medium, as compared with that on text, may reflect the expense and difficulty of constructing experimental narrative films. Specifically, although it is relatively easy to write experimental narrative texts, constructing narrative films requires relatively skilled actors and considerable technical knowledge. The present study does suggest that there are merits to conducting research in which existing films are used. Many researchers interested in studying this medium will not have the resources to construct experimentally generated films. However, we believe that this study suggests that despite the costs, creating experimentally controlled - most likely, shorter-films could be scientifically fruitful. With respect to the role of centrality in film comprehension, these shorter films could systematically vary the centrality of characters, while controlling for other factors. Furthermore, one could vary the centrality of characters both within a scene and at the level of the global plot. Theories of discourse comprehension vary in the extent to which they assume that readers monitor story constituents that are locally and globally related to the prior context (e.g., Graesser et al., 1994; McKoon \& Ratcliff, 1992). Such manipulations would allow one to further test theories of discourse understanding in the context of narrative film comprehension. This said, we be- lieve that experimentally generated films should engender aspects of naturalistic film, such as those explored here.

\section{REFERENCES}

Albrecht, J. E., O’Brien, E. J., Mason, R. A., \& Myers, J. L. (1995). The role of perspective in the accessibility of goals during reading. Journal of Experimental Psychology: Learning, Memory, \& Cognition, 21, 364-372.

Allbritton, D. W., \& Gerrig, R. J. (1991). Participatory responses in text understanding. Journal of Memory \& Language, 30, 603-626.

BAGgetT, P. (1979). Structurally equivalent stories in movie and text and the effect of the medium on recall. Journal of Verbal Learning \& Verbal Behavior, 18, 333-356.

Black, J. B., Turner, T. J., \& Bower, G, H. (1979). Point of view in narrative comprehension, memory, and production. Journal of Verbal Learning \& Verbal Behavior, 18, 187-198.

Bordwell, D. (1985). Narration in the fiction film. Madison: University of Wisconsin Press.

Bordwell, D., \& Thompson, K. (1993). Film art: An introduction. New York: McGraw-Hill.

Broccoli, A. R. (Producer), \& Gilbert, L. (Director) (1979). Moonraker [Film]. Available from CBS/Fox Video, Industrial Park Drive, Farmington Hills, MI 48024.

Coonan, J. R., Moder, M., Wizan, J. (Producers), \& Pollack, S. (DIRECTOR) (1972). Jeremiah Johnson [Film]. Available from Warner Brothers, 4000 Warner Boulevard, Burbank, CA 91522.

DE Wied, M., Zillmann, D., \& Ordman, V. (1994). The role of empathic distress in the enjoyment of cinematic tragedy. Poetics, 23, 91-106.

Fillmore, C. J. (1968). The case for case. In E. Bach \& T. Harms (Eds.), Universals in linguistic theory (pp. 1-90). New York: Holt, Rinehart \& Winston.

Fillmore, C. J. (1972). Some problems for case grammar. In R. J. O'Brien (Ed.), Report of the 22nd Annual Round Table Meeting on Linguistics and Language Studies (pp. 35-56). Washington, DC: Georgetown University Press.

Gerrig, R. J. (1996). Participatory aspects of narrative understanding. In R. J. Kreuz \& M. S. MacNealy (Eds.), Empirical approaches to literature and aesthetics (pp. 127-142). Norwood, NJ: Ablex.

Graesser, A. C. (1981). Prose comprehension beyond the word. New York: Springer.

Graesser, A. C., Bowers, C., Olde, B., \& Pomeroy, V. (1999). Who said what? Source memory for narrator and character agents in literary short stories. Journal of Educational Psychology, 91, 284-300.

Graesser, A. C., Bowers, C., Olde, B., White, K., \& Person, N. K. (1999). Who knows what: Propagation of knowledge among agents in a literary storyworld. Poetic, 26, 143-175.

Graesser, A. C., \& Clark, L. F. (1985). Structures and procedures of implicit knowledge. Norwood, NJ: Ablex.

Graesser, A. C., Singer, M., \& Trabasso, T. (1994). Constructing inferences during narrative text comprehension. Psychological Review, 101, 371-395.

Holland, N. N. (1992). Film response from eye to I: The Kuleshov experiment. In J. Gaines (Ed.), Classical Hollywood narrative: The paradigm wars (pp. 415-442). Durham, NC: Duke University Press.

Horton, W. S., \& RAPP, D. N. (2003). Out of sight, out of mind: Occlusion and the accessibility of information in narrative comprehension. Psychonomic Bulletin \& Review, 10, 104-110.

LonG, D. L., \& Golding, J. M. (1993). Superordinate goal inferences: Are they automatically generated during comprehension? Discourse Processes, 16, 55-73.

Lutz, M. F., \& Radvansky, G. A. (1997). The fate of completed goal information in narrative comprehension. Journal of Memory \& Language, 36, 293-310.

Magliano, J. P., Dijkstra, K., \& Zwann, R. (1996). Generating predictive inferences while viewing a movie. Discourse Processes, 22, 199-224.

Magliano, J. P., \& Graesser, A. C. (1991). A three-pronged method for studying inference generation in literary text. Poetics, 20, 193-232.

Magliano, J. P., Miller, J., \& ZwaAn, R. A. (2001). Indexing space and time in film understanding. Applied Cognitive Psychology, 15, 533-545. 
Magliano, J. P., \& Radvansky, G. A. (2001). Goal coordination in narrative comprehension. Psychonomic Bulletin \& Review, 8, 372-376.

Magliano, J. P., ZwaAn, R. A., \& Graesser, A. C. (1999). The role of situational continuity in narrative understanding. In S. R. Goldman \& $\mathrm{H}$. van Oostendorp, The construction of mental representations during reading (pp. 219-245). Mahwah, NJ: Erlbaum.

Mandler, J. M., \& Johnson, S. S. (1977). Remembrance of things parsed: Story structure and recall. Cognitive Psychology, 9, 111-151.

McKoon, G., \& Ratcliff, R. (1992). Inference during reading. Psychological Review, 99, 440-466.

Metz, C. (1982). The imaginary signifier: Psychoanalysis and the cinema. Bloomington: Indiana University Press.

Morrow, D. G. (1985). Prominent characters and events organize narrative understanding. Journal of Memory \& Language, 24, 304-319.

Myers, J. L., \& O'Brien, E. J. (1998). Accessing the discourse representation during reading. Discourse Processes, 26, 131-157.

Newtson, D. (1973). Attribution and the unit of perception of ongoing behavior. Journal of Personality \& Social Psychology, 28, 28-38.

ÖZyüreK, A., \& Trabasso, T. (1997). Evaluation during the understanding of narratives. Discourse Processes, 23, 305-335.

RAPP, D. N., \& GERRIG, R. J. (2002). Readers' reality-driven and plotdriven analyses in narrative comprehension. Memory \& Cognition, 30, 779-788.

Richards, E., \& Singer, M. (2001). Representation of complex goal structures in narrative comprehension. Discourse Processes, 31, 111135.

Sallin, R. (Producer), \& Meyer, N. (Director) (1982). Star Trek II: The Wrath of Khan [Film]. Available from Paramount Pictures, 5555 Melrose Avenue, Hollywood, CA 90038.

Schank, R. C., \& Abelson, R. (1977). Scripts, plans, and goals. Hillsdale, NJ: Erlbaum.

Schwan, S., Garsoffky, B., \& Hesse, F. W. (2000). Do film cuts facilitate the perceptual and cognitive organization of activity sequences? Memory \& Cognition, 28, 214-223.

ScotT Rich, S., \& TAYLOR, H. A. (2000). Not all narrative shifts function equally. Memory \& Cognition, 28, 1257-1266.

Stein, N. L., \& GLEnN, C. G. (1979). An analysis of story comprehension in elementary school children. In R. O. Freedle (Ed.), New directions in discourse processing (pp. 53-120). Norwood, NJ: Ablex.
SuH, S. Y., \& Trabasso, T. (1993). Inferences during reading: Converging evidence from discourse analysis, talk-aloud protocols, and recognition priming. Journal of Memory \& Language, 32, 279-300.

TAN, E. S. (1996). Emotions and the structure of narrative film. Mahwah, NJ: Erlbaum.

ThORNDYKe, P. W. (1977). Cognitive structures in comprehension and memory of narrative discourse. Cognitive Psychology, 9, 77-110.

Trabasso, T., \& Nickels, M. (1992). The development of goal plans of action in the narration of a picture story. Discourse Processes, $\mathbf{1 5}$, 249-275.

Trabasso, T., \& Sperry, L. (1985). Causal relatedness and importance of story events. Journal of Memory \& Language, 24, 595-611.

Trabasso, T., \& SuH, S. (1993). Understanding text: Achieving explanatory coherence through on-line inferences and mental operations in working memory. Discourse Processes, 16, 3-34.

Trabasso, T., \& van Den Broek, P. (1985). Causal thinking and the representation of narrative events. Journal of Memory \& Language, 24, 612-630.

Trabasso, T., van den Broek, P., \& Suh, S. (1989). Logical necessity and transitivity of causal relations in the representation of stories Discourse Processes, 12, 1-25.

VAn Den Broek, P., Risden, K., \& Husebye-Hartmann, E. (1995). The role of readers' standards for coherence in the generation of inferences during reading. In R. F. Lorch, Jr., \& E. J. O'Brien (Eds.), Sources of coherence in reading (pp. 353-373). Hillsdale, NJ: Erlbaum.

ZACKs, J. M., TVERsKy, B., \& IYER, G. (2001). Event structure in perception and conception. Psychological Bulletin, 127, 3-21.

ZillmanN, D. (1994). Mechanisms of emotional involvement with drama. Poetics, 23, 33-51.

Zwaan, R. A., Langston, M. C., \& Graesser, A. C. (1995). The construction of situation models in narrative comprehension: An eventindexing model. Psychological Science, 6, 292-297.

ZwaAn, R. A., \& Radvansky, G. A. (1998). Situation models in language comprehension and memory. Psychological Bulletin, 123, 162 185

(Manuscript received December 9, 2003; revision accepted for publication November 18, 2004.) 